\title{
Diseño de un modelo de gestión estratégico para el mejoramiento de la productividad y calidad
}

\section{Design of a strategic management model to improving productivity and} quality

Narcisa de Jesús Portalanza Molina. ${ }^{1}$, Vanessa Lorena Valverde González. ${ }^{2}$

Recibido: 15-05-2019 / Revisado: 18-06-2019 /Aceptado: 09-07-2019/ Publicado: 28-07-2019

\begin{abstract}
.
DOI: https://doi.org/10.33262/cienciadigital.v3i3.2.1.788

The aim of this research was to design a model of strategic management to improve productivity and quality in the Department of Engineering of "Commercial Technical Organization" Company. The development of the management model was applied, because of the shortcomings noted in the processes, diagnosing initial surveys through strategic, tactical, operational and commercial level situation. Internal processes were determined in conflict such as: customer service, sales, purchasing, training and production, which have been assessed by key indicators that have allowed them to raise strategic objectives, to be interpreted in the scorecard, under its four perspectives: financial, customer, internal processes, learning and growth. A matrix of indicators through the process map has been developed in order to advance a tab control to improve the performance of times in the duties, to achieve satisfaction. In developing the scorecard strategies to assist the fulfillment the established objectives, presenting indicators up $60 \%$ to $68 \%$, with an efficiency of $88 \%$ in the process, being determined so that the management model if it contributes to improving the productivity and quality of Engineering Department of the Company.
\end{abstract}

Keywords: Technology of industrial processes, management, scorecard, electrical projects, productivity

\section{Resumen}

El objetivo de la presente investigación es el diseño de un modelo de gestión estratégico para el mejoramiento de la productividad y calidad en el Departamento de Ingeniería

\footnotetext{
${ }^{1}$ Escuela Superior Politécnica de Chimborazo, Unidad de Admisión y Nivelación, Riobamba, Ecuador. narciza.portalanza@espoch.edu.ec

${ }^{2}$ Escuela Superior Politécnica de Chimborazo, Facultad de Mecánica, Carrera de Mantenimiento Industrial, Riobamba, Ecuador. v_valverde@espoch.edu.ec
} 
de la Empresa "Organización Técnica Comercial". El desarrollo del modelo de gestión se aplicó debido a las falencias encontradas en los procesos, diagnosticando su situación inicial a través de encuestas a nivel estratégico, táctico, operativo y comercial. Se determinó los procesos internos en conflicto como son: atención al cliente, ventas, compras, capacitación y producción, los cuales han sido evaluados por medio de indicadores claves, planteando objetivos estratégicos, para ser interpretados en el cuadro de mando integral, bajo sus cuatro perspectivas: financiera, del cliente, de procesos internos, de aprendizaje y crecimiento.

Se ha elaborado una matriz de indicadores a través del mapa de procesos con el fin de generar una ficha de control para la mejora de tiempos en la ejecución de obras, mientras que en el desarrollo del cuadro de mando integral se estableció estrategias que ayudarán al cumplimiento de objetivos, presentando un alza en los indicadores del $60 \%$ al 68\%, con una eficiencia del $88 \%$ en los procesos, determinándose así que el modelo de gestión contribuye a la mejora de la productividad y calidad del Departamento de Ingeniería.

Palabras claves: Tecnología de los procesos industriales, gestión, cuadro de mando integral, proyectos eléctricos, productividad.

\section{Introducción.}

En la presente investigación se va a desarrollar un modelo de gestión que tendrá un impacto positivo en el departamento de ingeniería de la empresa ORGATEC, permitiendo solucionar la problemática que este atraviesa, con el fin de bajar los costos en la ejecución de proyectos eléctricos y obtener la fidelidad de los clientes externos e internos a través de su nivel de satisfacción, utilizando estrategias que permitan solucionar problemas específicos como reprocesos, baja calidad, que son factores que afectan a la imagen del departamento y por ende de la empresa. ORGATEC “Organización Técnica Comercial” es una empresa dedicada a la generación de proyectos eléctricos desde su diseño hasta su construcción, siendo la falta de seriedad en la entrega de proyectos y los altos costos que se propone en las cotizaciones el problema central que tiene el departamento de ingeniería, esto se ha dado debido a que no existe una buena planificación, la comunicación es deficiente entre los colaboradores de los departamentos y la falta de profesionalismo por parte de algunos de los trabajadores del departamento de ingeniería.

Esta comunicación debe incluir una descripción de estas y los objetivos que se pretenden lograr, incluyendo el mensaje de que cada persona tiene una responsabilidad personal sobre la calidad y se espera que cumpla con estas políticas y procedimientos. (CT, 2017).

Si el problema persiste las entregas de los proyectos eléctricos tendrán retrasos más significativos, insatisfacción en el cliente generando así la pérdida de estos, reprocesos en el ensamblaje de tableros, etc. 
Además del incremento de la competencia por la emergencia de actores con ventajas en costes, la globalización también permite mejorar la productividad a través del aprovechamiento de la fragmentación del proceso productivo y la posibilidad de externalizar aquellas fases que sean menos productivas (Borrego, 2014).

La calidad no depende "de la prima con que se miran las cosas", sino de la habilidad de la persona y puede alcanzarse pragmáticamente con sólo eliminar las prácticas generadoras de despilfarro; entonces si es posible duplicar los beneficios en un año. (Salvio, 2015)

Como objetivo se plantea determinar los indicadores claves que van a formar parte del cuadro de mando integral para el desarrollo del modelo de gestión, analizar los procesos a través de un mapeo y establecer estrategias para el desarrollo del modelo de gestión. La hipótesis planteada como el diseño de un modelo de gestión estratégico mejora la calidad y productividad de la empresa ORGATEC, será comprobada a través del avance de la investigación.

\section{Metodología}

La metodología por emplear en el desarrollo del modelo de gestión es la investigación explicativa, debido a que permite explicar el funcionamiento de los procesos y como se puede mejorar su procedimiento para establecer los parámetros de cambio.

ORGATEC, es una empresa que se dedica a la comercialización de material eléctrico de media y baja tensión, además ofrece servicios de desarrollo y ejecución de proyectos de ingeniería eléctrica.

La población de estudio estará conformada por todos los colaboradores internos y externos que laboran en el departamento de ingeniería de la empresa como son linieros, técnicos, secretaria e ingenieros, siendo un conjunto total de 23 personas incluido el señor gerente. Por lo tanto, la población objetivo será de 23 colaboradores. Podemos establecer que el cálculo del tamaño de la muestra resulta ser uno de los aspectos claves en la fase previa de cualquier investigación científica o de mercado, ya que con esta es posible determinar el grado de credibilidad que le podemos asignar a los resultados de la investigación.

Además, al elegir un buen tamaño de muestra y una técnica adecuada de muestreo, implícitamente hemos reunido información que cumple las características de validación, confiabilidad, representación a un costo mínimo. (Gutiérrez, 2014)

Para el desarrollo del diagnóstico se toma en cuenta solo la estructura del departamento de ingeniera, el cual se muestra en la siguiente figura: 
Figura 1. Organigrama del departamento de ingeniería de ORGATEC 2016

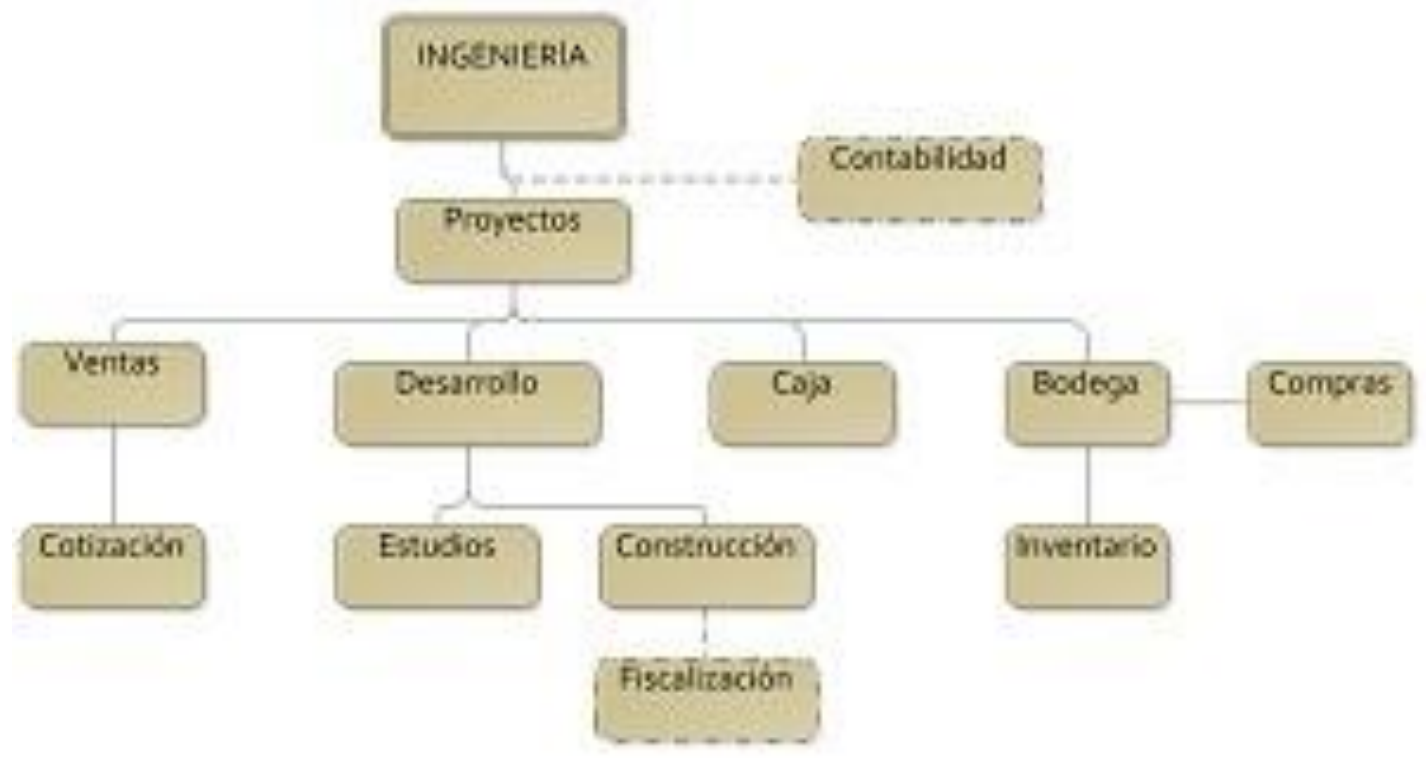

Fuente: ORGATEC

La energía eléctrica permite el vivir en comodidad, además de que es la base fundamental en un proceso de transformación de productos. Existen estaciones de distribución de energía eléctrica para todo el Ecuador, accediendo así que a cada hogar llegue la energía eléctrica, por medio de conductores eléctricos que están divididos en media tensión y baja tensión. En primer lugar, para que los equipos eléctricos realicen su función será necesaria una instalación eléctrica, que partiendo de un punto de suministro (acometida) permitirá distribuir dicha energía y ser consumida por los diferentes equipos conectados a esa instalación. (Jiménez, 2015)

Esto ha permitido generar fuentes de trabajo para empresas privadas, las mismas que están dedicadas a brindar el servicio que la institución pública no puede ofrecer debido a la gran demanda que existe en el diseño y construcción de proyectos eléctricos, los mismos que deben seguir el siguiente proceso: pedido del cliente, inspección, elaboración del presupuesto, elaboración del diseño, aprobación EERSA, fiscalización, solicitud de autorización para desconexión, solicitud de fecha para desconexión, publicación de fecha de desconexión con 48 horas de anticipación. Toda desconexión se hace con la presencia de un fiscalizador en la fecha dada, se realiza el trabajo, se conecta con presencia del fiscalizador, terminada la obra se elabora una memoria de construcción y acta de finalización de la obra, se certifica por planificación de la EERSA, se sube la información certificada al GO DATA BASE de la EERSA, se realiza el pago correspondiente a la energización, previo al informe del fiscalizador, se realiza pruebas (medición de voltajes), se firman oficios y actas finales entre gerente y fiscalizador (provisional), comercialización permite la compra del medidor, se firman oficios y actas finales entre gerente y fiscalizador (definitiva después de 6 meses). 
Para llevar la energía eléctrica desde la generación a los consumos, se utilizan canalizaciones tanto en alta como en baja tensión, dónde se destacan la aérea y la subterránea. A la hora de considerar las características de estas canalizaciones, debemos tener en cuenta tanto la normativa vigente como las recomendaciones dadas por las compañías eléctricas. (Trashorras, 2016)

La construcción de proyectos eléctricos se la puede describir de forma general, debido a la necesidad del cliente, no todos los proyectos son iguales, por lo tanto se realiza un sub proceso para identificar los pasos que se siguen, descritos de la siguiente forma: verificar la parte de construcción civil terminada, previa la aprobación del fiscalizador, se realiza la parte que no necesita desconexión como es: excavación huecos para plantación de postes BT, MT y los bloques de anclaje, arada de postes y tapada de bloques de anclaje, armada de estructuras de MT y BT (según el circuito sea trifásico, bifásico o monofásico), puesta de tensores a sus anclajes y en los diferentes postes, tendida de línea de MT y BT, colocación de fuentes visibles (seccionadores), levantamiento de finalización de la obra (medición, conteo de materiales y puntos GPS), el día de desconexión, se realizan los siguientes pasos: se desconecta el seccionador del ramal principal, se puentea las líneas para alimentar al nuevo seccionador que alimenta la nueva red, se energiza en Media Tensión y en Baja Tensión.

Además, la empresa realiza el diseño y construcción de tableros eléctricos, el mismo que sirve para la automatización de maquinaria industrial. El proceso de elaboración de los tableros sigue el procedimiento descrito a continuación, a partir de la necesidad del cliente.

Primero se realiza la inspección para la toma de datos (cargas), elabora el diagrama de fuerza y de control para cubrir la necesidad del cliente, se elabora un listado de materiales, el mismo que se envía para la elaboración de la cotización de la obra, después de la aprobación de la cotización y el acuerdo de la forma de pago del cliente. se procede al ensamblaje, se realiza pruebas de funcionamiento sin cargas, se instala en el lugar destinado, previo la elaboración de una malla de puesta a tierra, se conecta y se realiza pruebas de funcionamiento final.

Para el cálculo de la productividad operativa se recabó información referente al año 2015, en donde se tiene datos operativos como producción realizada (cotizaciones de proyectos aprobados) por periodos mensuales, la producción no realizada o rechazada por motivos de costo o de tiempos (cotizaciones rechazadas), los tiempos operativos del sistema y el grado en el que se utilizaron en relación con lo programado. 
Tabla 1. Productividad de la empresa ORGATEC 2015

\begin{tabular}{|c|c|c|c|c|c|c|c|c|}
\hline$\sum_{\Sigma}^{e}$ & 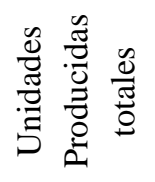 & 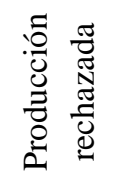 & 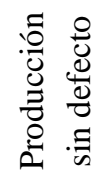 & 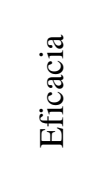 & 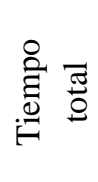 & 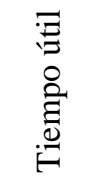 & 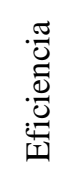 & 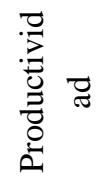 \\
\hline ene-15 & 5 & 1 & 4 & $80 \%$ & 33 & 30 & $91 \%$ & $73 \%$ \\
\hline feb-15 & 3 & 0 & 3 & $100 \%$ & 24 & 21 & $88 \%$ & $88 \%$ \\
\hline mar-15 & 5 & 0 & 5 & $100 \%$ & 34 & 31 & $91 \%$ & $91 \%$ \\
\hline abr-15 & 5 & 1 & 4 & $80 \%$ & 33 & 30 & $91 \%$ & $73 \%$ \\
\hline may-15 & 3 & 0 & 3 & $100 \%$ & 24 & 20 & $83 \%$ & $83 \%$ \\
\hline jun-15 & 4 & 0 & 4 & $100 \%$ & 29 & 26 & $90 \%$ & $90 \%$ \\
\hline jul-15 & 4 & 1 & 3 & $75 \%$ & 30 & 27 & $90 \%$ & $68 \%$ \\
\hline ago-15 & 3 & 0 & 3 & $100 \%$ & 25 & 21 & $84 \%$ & $84 \%$ \\
\hline sep-15 & 4 & 0 & 4 & $100 \%$ & 29 & 26 & $90 \%$ & $90 \%$ \\
\hline oct-15 & 3 & 1 & 2 & $67 \%$ & 24 & 21 & $88 \%$ & $58 \%$ \\
\hline nov-15 & 3 & 0 & 3 & $100 \%$ & 24 & 22 & $92 \%$ & $92 \%$ \\
\hline dic-15 & 4 & 1 & 3 & $75 \%$ & 30 & 26 & $87 \%$ & $65 \%$ \\
\hline \multicolumn{4}{|c|}{ Promedios totales: } & $90 \%$ & 28,25 & 25,08 & $89 \%$ & $79 \%$ \\
\hline
\end{tabular}

Fuente: Archivo de la empresa

La eficacia de la productividad para el periodo 2015 es del 90\%, demostrando así que el 10\% de proyectos son rechazados por el costo de la ejecución o por el incumplimiento en tiempos de entrega, así mismo, se tiene que la eficiencia es del 89\%, es decir que al ejecutar las obras se pierde en tiempos el $11 \%$, tiempo que pueden ser por la mala distribución del material o mala elaboración del listado de elementos o por la falta de revisión de herramientas de trabajo, entre otras causas. La productividad resultante del sistema es del 79\%, el cual indica que se puede mejorar, al adoptar el sistema de gestión.

La esencia de la mejora de la productividad es un ciclo interminable de buscar y encontrar las causas de fallas o defectos, y arreglarlas o erradicarlas; o, por lo menos, una vez identificadas, atenuar sus consecuencias, entendiendo por falla o defecto cualquier resultado del proceso. (Fonseca, 2015)

El índice de productividad obtenido es del 13\%, esto indica que la empresa está produciendo correctamente, es decir no está trabajando con pérdidas. Ningún empresario invertirá su dinero en una actividad que le diera pérdidas.

Un empresario puede tener mala suerte o un criterio equivocado a la hora de invertir, pero ninguno invierte para perder. Por el contrario, cada uno buscará que su inversión sea lo más rentable posible. Para ello tratará de que su empresa produzca la mayor cantidad de bienes o servicios en el menor tiempo posible. Es decir, buscará incrementar la productividad. (Nardulli, 2015) 
Tabla 2. Productividad multifactorial de la empresa ORGATEC 2015

\begin{tabular}{cccc}
\hline \hline Mes & Costo producción & Costo total & Productividad \\
\hline ene-15 & 3117,84 & 2642,24 & 1,18 \\
feb-15 & 15029,61 & 13526,65 & 1,11 \\
mar-15 & 13372,38 & 11855,72 & 1,13 \\
abr-15 & 5137,07 & 4576,49 & 1,12 \\
may-15 & 8940,59 & 8046,53 & 1,11 \\
jun-15 & 11431,87 & 10267,47 & 1,11 \\
jul-15 & 467,53 & 432,9 & 1,08 \\
ago-15 & 11290,2 & 10161,18 & 1,11 \\
sep-15 & 4418,43 & 3976,59 & 1,11 \\
oct-15 & 2109,18 & 1898,26 & 1,11 \\
nov-15 & 339,57 & 287,77 & 1,18 \\
dic-15 & 183,07 & 151,3 & 1,21 \\
& $\mathbf{7 5 8 3 7 , 3 4}$ & $\mathbf{6 7 8 2 3 , 1}$ & $\mathbf{1 , 1 3}$ \\
\hline \hline
\end{tabular}

Fuente: Archivo de la empresa

El modelo comprende dos fases importantes la estrategia y la estructura de la organización. En la primera fase se determina el patrón de la organización y se establece las acciones a realizar, especificando los pasos a seguir en cuanto a la razón de la empresa como es la Misión, Visión y objetivos, que deberán ser planteados para gestionar el modelo. En la segunda fase se hará énfasis al enfoque de procesos y se establecerán todos los pasos necesarios para su desarrollo y el control de indicadores a través del control estadístico de procesos.

Tabla 3. Lista de procesos internos del departamento de ingeniería.

\begin{tabular}{l}
\hline PROCESOS INTERNOS ORGATEC \\
\hline Atención al cliente \\
Proceso de construcciones \\
Proceso de ventas \\
Ejecución de proyectos \\
Proceso de compras \\
\hline \hline
\end{tabular}

Fuente: Archivo de la empresa

Para obtener un enfoque basado en procesos es necesario determinar cuáles son los procesos que deben formar parte del sistema, es decir los que van a ser parte de la estructura de procesos del sistema, por lo tanto, se muestra a continuación los procesos que actualmente tiene el departamento de ingeniería de la empresa. 
El principal punto de interés se resume en una sola frase: mejoramiento de la calidad y efectos de su aplicación en procesos de producción, ya sea de bienes o de servicios desde un punto de vista mucho más económico que contable. (Toro, 2016)

En este punto se evaluará si el proceso tiene relación con la calidad del producto, ya sea de forma positiva o negativa, siendo evaluado mediante ponderaciones: Muy alto (5), alto (4), Medio (3), Bajo (2) y Muy Bajo (1). Como se muestra en la tabla 4.

Tabla 4. Matriz de relación de procesos

\begin{tabular}{|c|c|c|c|c|}
\hline Proceso & $\begin{array}{l}\text { Satisfacción del } \\
\text { cliente }\end{array}$ & $\begin{array}{l}\text { Impacto del } \\
\text { proceso }\end{array}$ & $\begin{array}{l}\text { Efectos en la } \\
\text { calidad }\end{array}$ & TOTAL \\
\hline Atención al cliente & 4 & 3 & 4 & 48 \\
\hline Compras & 5 & 4 & 5 & 100 \\
\hline Ventas & 5 & 4 & 4 & 80 \\
\hline Contabilidad & 4 & 5 & 2 & 40 \\
\hline Facturación & 3 & 3 & 3 & 27 \\
\hline Sistemas de información & 3 & 4 & 3 & 36 \\
\hline Capacitación & 5 & 4 & 2 & 40 \\
\hline Entrega de obras & 5 & 4 & 4 & 80 \\
\hline Mejora continua & 3 & 3 & 2 & 18 \\
\hline Gestión de bodegas & 5 & 5 & 4 & 100 \\
\hline Gestión de los recursos & 4 & 4 & 3 & 48 \\
\hline Gestión del personal & 4 & 3 & 4 & 48 \\
\hline Gestión financiera & 5 & 3 & 4 & 60 \\
\hline Gestión de riesgos laborales & 3 & 4 & 4 & 48 \\
\hline Gestión Medioambiental & 3 & 3 & 4 & 36 \\
\hline Investigación del Mercado & 3 & 3 & 2 & 18 \\
\hline Gestión por procesos & 4 & 4 & 3 & 48 \\
\hline Pedidos & 5 & 4 & 4 & 80 \\
\hline Construcciones & 4 & 5 & 5 & 100 \\
\hline Sistema de gestión de calidad & 5 & 4 & 4 & 80 \\
\hline Investigación y desarrollo & 2 & 2 & 2 & 8 \\
\hline Jurídico / Legal & 2 & 2 & 2 & 8 \\
\hline Mantenimiento de equipos & 3 & 4 & 4 & 48 \\
\hline Política de Talento Humano & 3 & 3 & 2 & 18 \\
\hline Talento Humano & 5 & 3 & 4 & 60 \\
\hline Ensamblaje & 5 & 5 & 4 & 100 \\
\hline Entrega & 4 & 2 & 2 & 16 \\
\hline
\end{tabular}

Fuente: Matriz de relación de procesos

A través de la matriz de relación de procesos, se han identificado los procesos claves, los cuales serán los de mayor puntaje, obtenido en la valoración otorgada por la administración de la empresa. Estos procesos permitirán desplegar las estrategias y objetivos de la organización a largo plazo, la descripción de estos se muestra en la siguiente tabla: 
Tabla 5. Procesos clave

\begin{tabular}{ll}
\hline \hline \multicolumn{1}{c}{ Proceso } & \multicolumn{1}{c}{ Actividades principales } \\
\hline Compras & $\begin{array}{l}\text { Búsqueda de nuevos proveedores, evaluación de proveedores, } \\
\text { negociación de precios, asignación de pedidos. } \\
\text { Ventas }\end{array}$ \\
Entrega de obras & Coordina logística externa \\
Gestión de bodegas & Almacena materiales y tableros terminados \\
Pedidos & $\begin{array}{l}\text { Gestión de ofertas, aceptación de pedidos, aprovisionamientos, } \\
\text { logística de facturación, cobros, seguimientos. }\end{array}$ \\
Construcciones & $\begin{array}{l}\text { Planifica los recursos necesarios para llevar a cabo la programación del } \\
\text { ensamblaje, verifica stock de material eléctrico. }\end{array}$ \\
Ensamblaje & $\begin{array}{l}\text { Recepta órdenes de trabajo, programa logística interna, realiza el } \\
\text { proceso de ensamblaje. }\end{array}$ \\
Controla especificaciones técnicas de los materiales a utilizar en la \\
\hline \hline
\end{tabular}

Fuente: Matriz de relación de procesos

Esta recomposición de actividades tiene un efecto directo de incremento de la productividad que está asociada con un cambio de composición de actividades en el que se sustituyen, en general, procesos menos eficientes por otros más eficientes. (Heizer, 2015).

Los procesos estratégicos serán: las comunicaciones internas, comunicación con el cliente, marketing, diseño y revisión del sistema, planificación estratégica y diseño de planes de estudio. Los procesos estratégicos serán: Capacitación, compras, auditorías internas, informática.

Cada uno de los procesos serán analizados a través de diagramas de flujo, con la finalidad de mostrar de forma detallada las actividades y responsabilidades que debe tener cada uno de los colaboradores de la empresa, según la actividad que desarrolla.

Una de las herramientas que nos ayudan a entender las complejidades del diseño y rediseño del proceso, es el diagrama de flujo, un esquema o dibujo el movimiento de materiales, productos o personas. (Render, 2014)

Este proceso tiene como objetivo cubrir la necesidad del cliente, permite establecer un acuerdo con la forma de pago, además realiza un análisis del cliente, si es nuevo se le ayuda con información técnica para que en un futuro se convierta en cliente potencial. 
Figura 2. Diagrama de flujo del proceso de Ventas

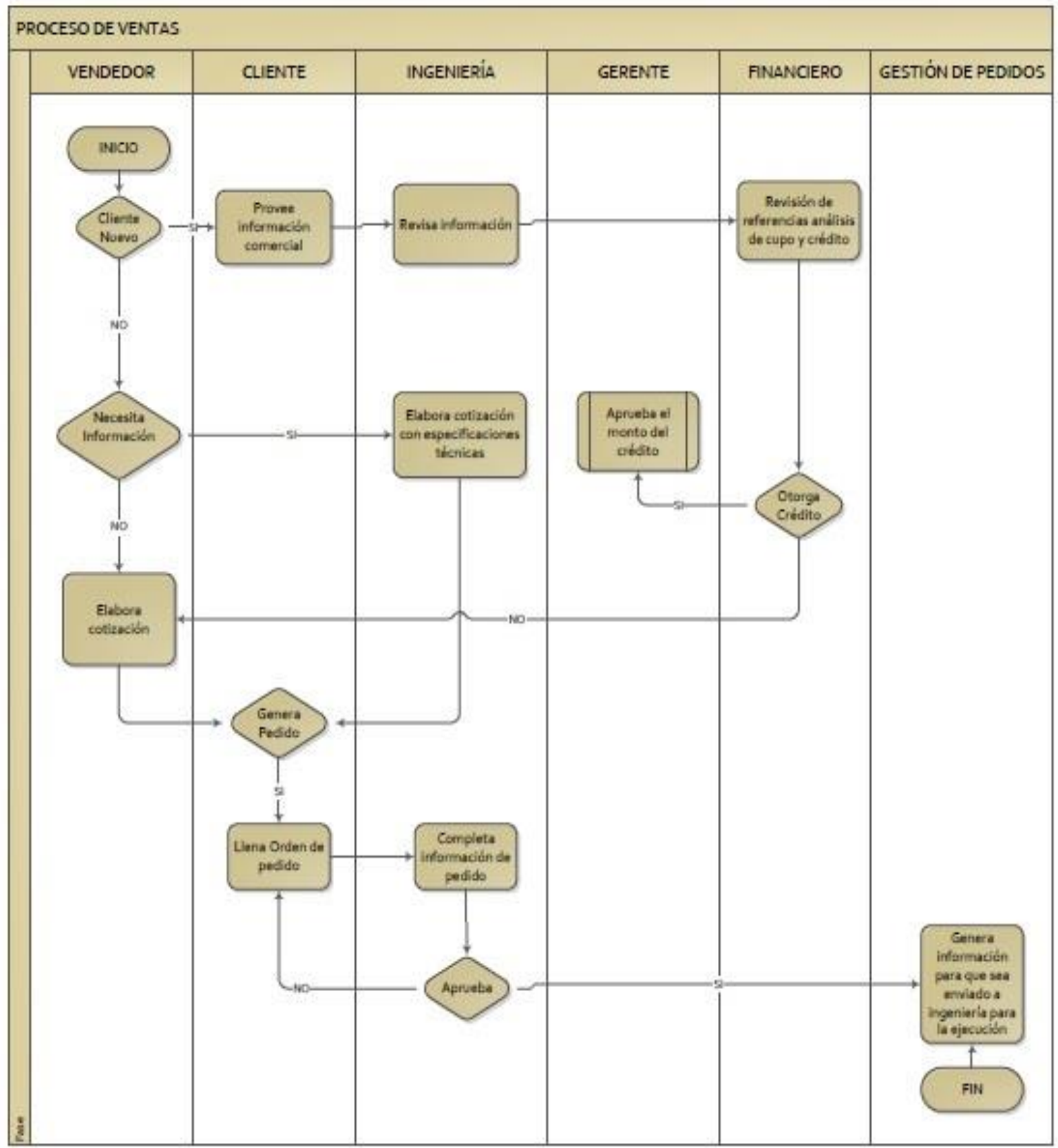

Fuente: ORGATEC

Proceso de atención al cliente: Este proceso permite identificar la necesidad del cliente y la posibilidad de que sea atendido de forma eficiente con información técnica que le permite satisfacer sus dudas y cubrir todas sus expectativas. 
Figura 3. Diagrama de flujo del proceso de Atención al Cliente

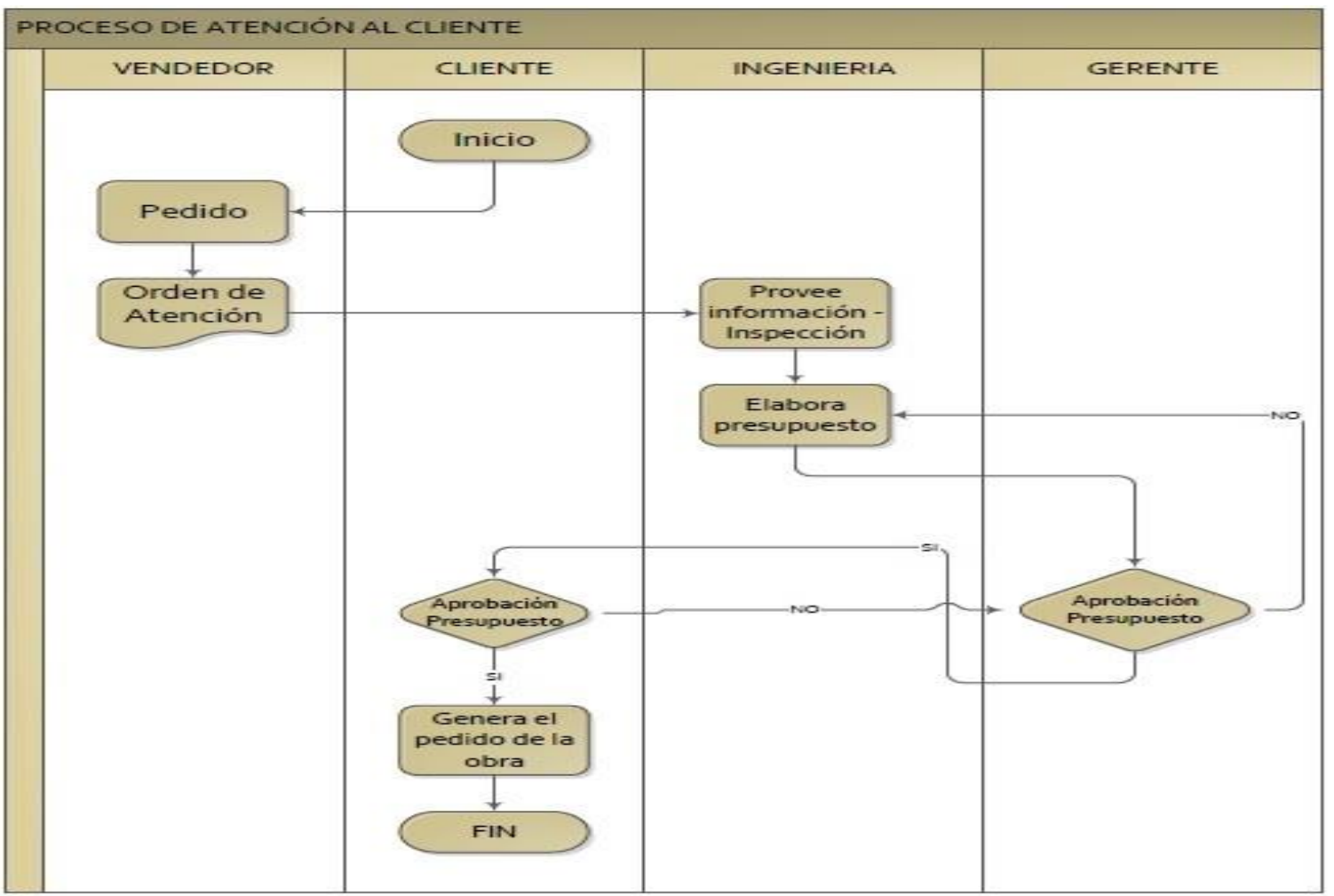

Fuente: ORGATEC

Este proceso permite definir si la obra se va a realizar o no, si la disponibilidad de materiales está completa o si se debe generar una orden de compra y si los materiales eléctricos cumplen o no con las especificaciones solicitadas por el cliente.

Figura 4. Diagrama de flujo del proceso Construcciones

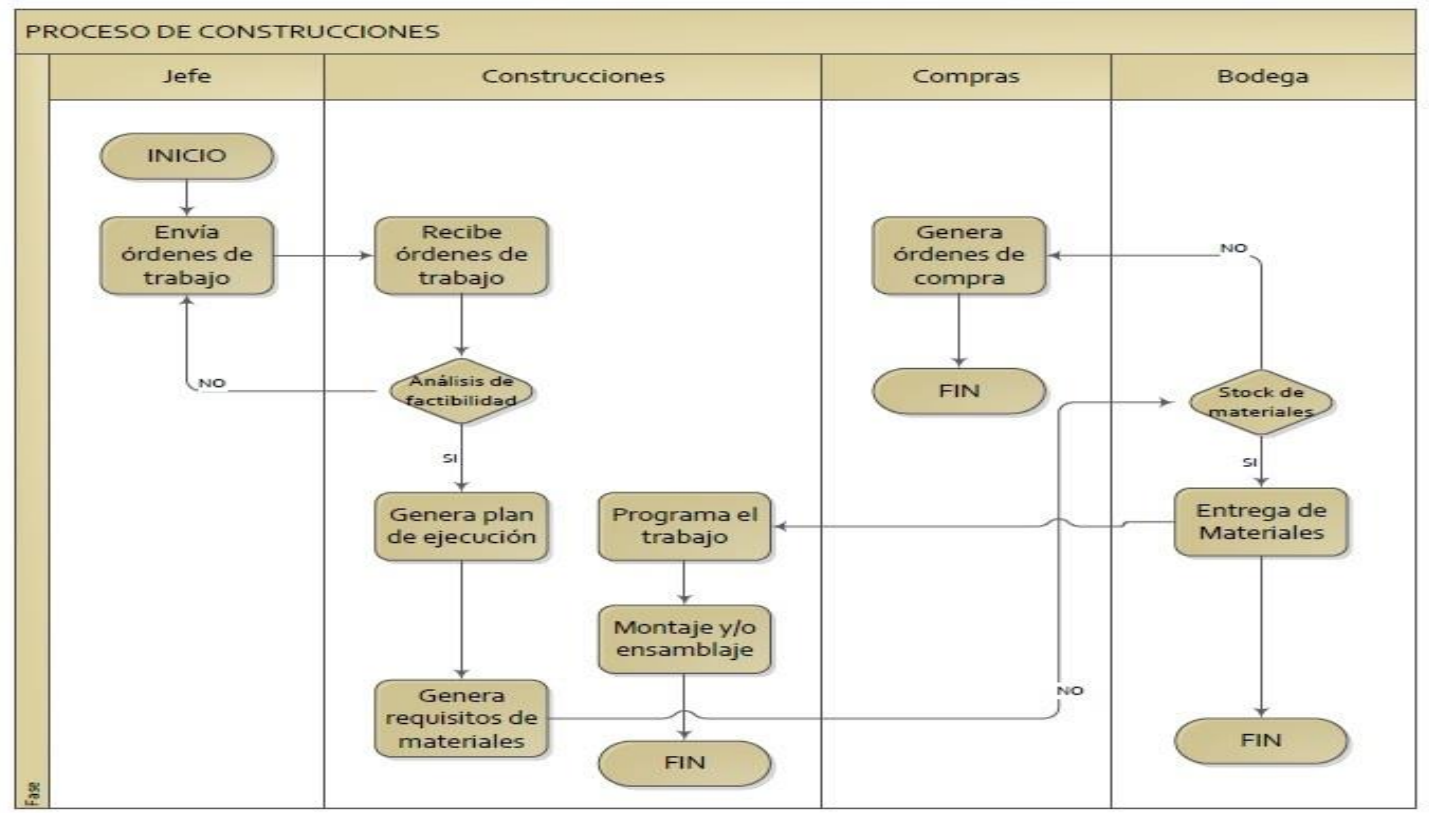

Fuente: ORGATEC 
Este proceso permite conocer de forma general como es el proceso de ejecución de proyectos eléctricos, además explica la forma como debe procederse a la aprobación de diseños en la EERSA, la subida de información al sistema de la EERSA, realización de pruebas de funcionamiento y entrega de la obra finalizada.

Figura 5. Diagrama de flujo del proceso de Construcciones

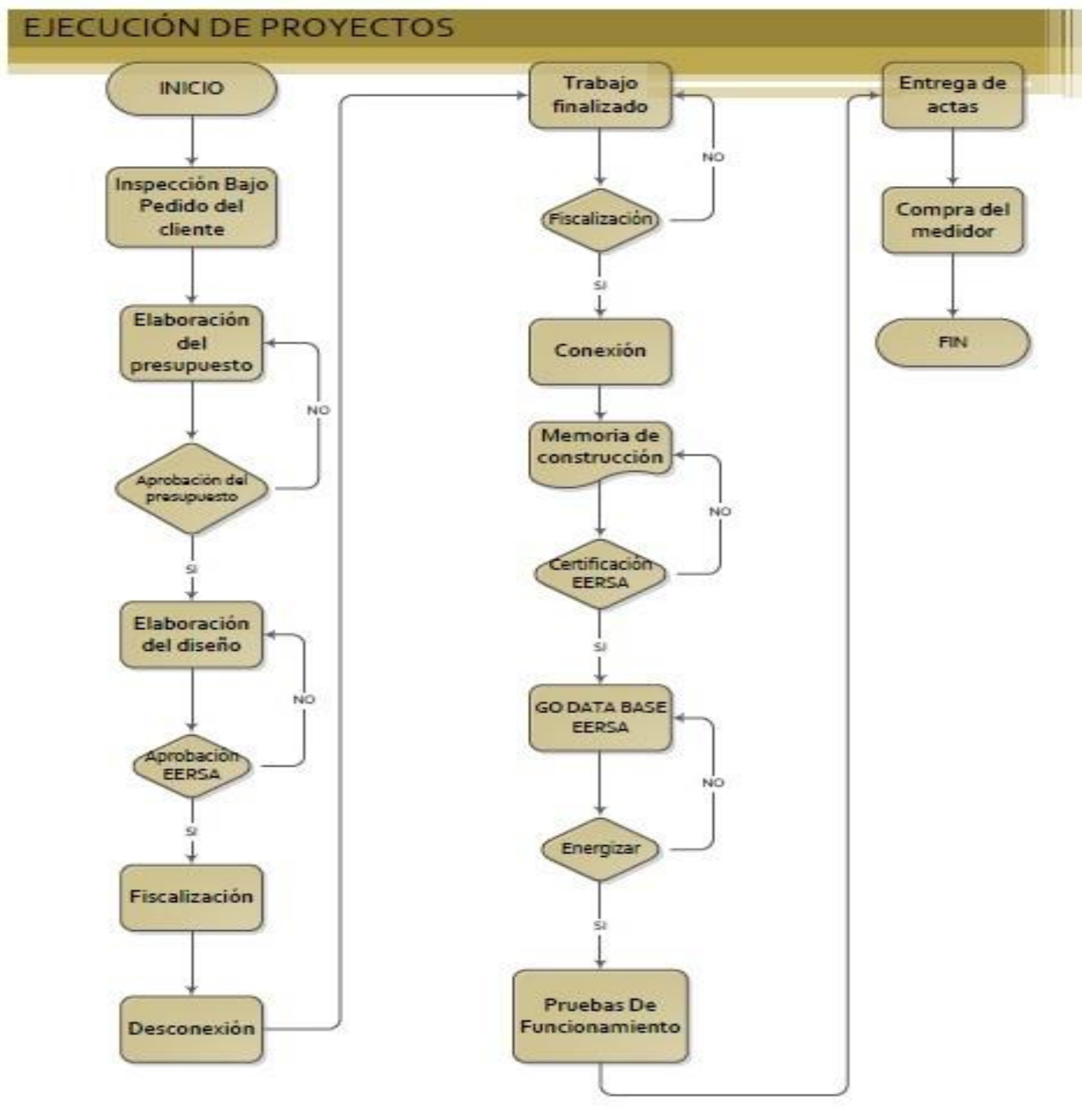

\section{Fuente: ORGATEC}

Establecidos los objetivos estratégicos en cada una de las perspectivas, se presenta el cuadro de mando integral con el análisis de sus indicadores a manera de semáforo demostrando su valor real y la meta a alcanzar. Los objetivos y las medidas del Cuadro de Mando Integral son algo más que una colección de indicadores de actuación financiera y no financiera; se derivan de un proceso vertical impulsado por el objetivo y la estrategia de la unidad de negocio. Para dar cumplimiento a los objetivos estratégicos planteados para el departamento de ingeniería de la empresa, es necesario un plan de acción que permita contar con la 
siguiente información: la estrategia a aplicarse, la forma mediante la cual se cumplirá la estrategia, el responsable de cumplir las actividades, los recursos que se necesitarán, el tiempo programado para cumplir la estrategia y el indicador que permita controlar el desempeño.

Tabla 6. Indicadores cuadro de mando integral

PERSPECTIVA INDICADOR

REAL META

FINANCIERA

Eficiencia en ventas

Rotación del stock de ítems en inventario

Rechazo a proveedores

Nivel de inventarios

Porcentaje de gastos financieros

Satisfacción del cliente

CLIENTE

PROCESOS

INTERNOS
Producto conforme

Proyectos entregados de acuerdo con lo programado

Desviación de desperdicio frente a presupuesto

Eficiencia del manejo de mano de obra

Costo por resultado de ensamblaje

Tasa de construcciones
$90 \% \quad 94 \%$

$20 \% \quad 22 \%$

$25 \% \quad 13 \%$

$42 \% \quad 30 \%$

$36 \% \quad 33 \%$

$80 \% \quad 100 \%$

$94 \% \quad 95 \%$

$75 \% \quad 100 \%$

$2 \% \quad 1,5 \%$

$89 \% \quad 91 \%$

$84 \% \quad 90 \%$

$75 \% \quad 100 \%$

Compras de materiales efectuadas dentro del tiempo programado

$20 \% \quad 25 \%$

Porcentaje de averías de vehículos

$25 \% \quad 0 \%$

Cumplimiento de auditorías internas

$80 \% \quad 100 \%$

Nivel de cumplimiento

$89 \% \quad 100 \%$

Capacitación

$77 \% \quad 100 \%$

APRENDIZAJE Y

$80 \% \quad 100 \%$

Índice de gravedad SSO

Fuente: Indicadores cuadro de mando integral 
Tomando en cuenta que la perspectiva financiera está relacionada con la estrategia de la empresa en cuanto a crecimiento y reducción de costos en la productividad, para dar cumplimiento a la misión y visión, se tiene las siguientes etapas a analizar: el crecimiento y diversificación de los ingresos, reducción de costos y mejora de la productividad, utilización de los activos y estrategia de inversión.

La perspectiva del cliente permite a la empresa identificar los segmentos de cliente y mercado en el que se puede competir. Permite, además, identificar y medir las propuestas de valor agregado siendo el indicador clave la satisfacción del cliente.

La perspectiva de procesos permite a la administración de la empresa identificar los procesos críticos que impiden el cumplimiento de los objetivos. Se recomienda definir una cadena de valor de los procesos con el fin de obtener un proceso innovador desarrollando nuevas soluciones para cubrir la necesidad del cliente, para después aplicar procesos productivos que identifiquen las expectativas del cliente y por último tener un proceso de servicio de post venta que añada valor a los servicios que reciben los clientes.

La perspectiva de aprendizaje y crecimiento permite que se alcancen los objetivos de las perspectivas anteriores, debido a que se hace énfasis en las inversiones a futuro.

Las capacidades de los empleados deben ser consideradas la estrategia más importante para la mejora de los procesos internos, debido a que están en contacto con los clientes y son la imagen de la empresa. Es importante considerar los siguientes aspectos: Satisfacción del empleado, Retención del empleado, Productividad del empleado.

La capacidad de alcanzar las metas de los objetivos financieros depende de la eficacia de los procesos internos, los mismos que se fundamentan en la capacidad y motivación que tiene el empleado. Por lo tanto, los indicadores del aprendizaje organizacional provienen de los empleados, los sistemas y el equipamiento de la empresa.

Realizando un análisis de eficiencia entre los valores reales y los valores meta de los indicadores mostrados en la tabla 10-4, se establece que el promedio de los indicadores reales es del $60 \%$, mientras que de los indicadores meta es del $68 \%$, por lo tanto, la eficiencia es del $88 \%$, se puede decir entonces que el modelo de gestión si aporta a la mejora de la productividad y calidad del departamento de ingeniería de la empresa ORGATEC.

Para llegar a cumplir con esta meta es necesario establecer cambios en el proceso de atención al cliente, el proceso de inspecciones, el proceso de compras, optimizando los tiempos a través de la mejora de estos.

La empresa debe invertir en su infraestructura, nuevos equipos, personal, sistemas y procedimientos, con el fin de alcanzar sus objetivos. 
Tabla 7. Matriz de resultados

\begin{tabular}{|c|c|c|c|c|c|}
\hline $\begin{array}{l}\text { Objetivo } \\
\text { Estratégico }\end{array}$ & $\begin{array}{c}\text { Forma de aplicación } \\
\text { del objetivo }\end{array}$ & Indicador & Método de Cálculo & Frecuencia & $\begin{array}{c}\text { Resultado } \\
\text { Esperado } \\
\%\end{array}$ \\
\hline $\begin{array}{l}\text { Maximizar } \\
\text { rentabilidad }\end{array}$ & $\begin{array}{l}\text { Mejorar el tiempo del } \\
\text { proceso de ventas }\end{array}$ & $\begin{array}{l}\text { Eficiencia en } \\
\text { ventas }\end{array}$ & $\begin{array}{l}\text { Obras realizadas por } \\
\text { semana/Obras perdidas } \\
\text { por semana } * 100\end{array}$ & Mensual & $94 \%$ \\
\hline $\begin{array}{l}\text { Mejorar } \\
\text { capital de } \\
\text { trabajo }\end{array}$ & $\begin{array}{l}\text { Invertir en material } \\
\text { eléctrico de mayor } \\
\text { rotación }\end{array}$ & $\begin{array}{l}\text { Rotación del } \\
\text { stock }\end{array}$ & $\begin{array}{l}\text { Consumo de ítems } \\
\text { industriales/Valor del } \\
\text { stock de ítems }\end{array}$ & Mensual & $22 \%$ \\
\hline $\begin{array}{l}\text { Asegurar } \\
\text { negociación } \\
\text { con } \\
\text { proveedores }\end{array}$ & $\begin{array}{l}\text { Hacer una selección } \\
\text { eficaz de proveedores }\end{array}$ & $\begin{array}{l}\text { Rechazo a } \\
\text { proveedores }\end{array}$ & $\begin{array}{l}\text { Compras } \\
\text { rechazadas/Compras } \\
\text { Aceptadas }\end{array}$ & Mensual & $13 \%$ \\
\hline $\begin{array}{l}\text { Reducir } \\
\text { costos }\end{array}$ & $\begin{array}{l}\text { No almacenar material } \\
\text { sin rotación }\end{array}$ & $\begin{array}{l}\text { Nivel de } \\
\text { inventarios }\end{array}$ & $\begin{array}{l}\text { Costo de } \\
\text { Inventario/Ventas } \\
\text { netas*100 }\end{array}$ & Mensual & $30 \%$ \\
\hline $\begin{array}{l}\text { Obtener } \\
\text { financiamient } \\
\text { o de } \\
\text { proyectos de } \\
\text { inversión y } \\
\text { crédito con } \\
\text { proveedores }\end{array}$ & $\begin{array}{l}\text { Medir el impacto de } \\
\text { gastos de } \\
\text { administración y } \\
\text { ventas }\end{array}$ & $\begin{array}{l}\text { Porcentaje de } \\
\text { gastos } \\
\text { financieros }\end{array}$ & $\begin{array}{l}\text { (Gastos financieros/Total } \\
\text { Ventas) } * 100\end{array}$ & Semestral & $33 \%$ \\
\hline $\begin{array}{l}\text { Mejorar el } \\
\text { nivel de } \\
\text { satisfacción } \\
\text { del cliente }\end{array}$ & $\begin{array}{l}\text { Atención eficaz, } \\
\text { cordial y rápida }\end{array}$ & $\begin{array}{l}\text { Satisfacción } \\
\text { del cliente }\end{array}$ & $\begin{array}{l}\text { Tabulación de la } \\
\text { percepción del cliente } \\
\text { sobre el servicio recibido }\end{array}$ & Semestral & $10 \%$ \\
\hline $\begin{array}{l}\text { Mejorar los } \\
\text { procesos de } \\
\text { desarrollo de } \\
\text { nuevos } \\
\text { servicios y } \\
\text { uso de } \\
\text { tecnologías }\end{array}$ & $\begin{array}{l}\text { Mejorar los procesos } \\
\text { de entrega de bodega y } \\
\text { el uso de equipos } \\
\text { tecnológicos }\end{array}$ & $\begin{array}{l}\text { Producto } \\
\text { conforme }\end{array}$ & $\begin{array}{l}\text { ((Productos suministrados } \\
\text { - productos devueltos) } \\
\text { /productos suministrados) } \\
* 100\end{array}$ & Mensual & $95 \%$ \\
\hline $\begin{array}{l}\text { Cumplir con } \\
\text { los } \\
\text { procedimient } \\
\text { os } \\
\text { planificados y } \\
\text { minimizar los } \\
\text { reprocesos }\end{array}$ & $\begin{array}{l}\text { Realizar los } \\
\text { procedimientos de los } \\
\text { procesos de ejecución } \\
\text { sin demoras }\end{array}$ & $\begin{array}{l}\text { Producto } \\
\text { entregado de } \\
\text { acuerdo con lo } \\
\text { programado }\end{array}$ & $\begin{array}{l}\text { Total de obras que } \\
\text { cumplen las } \\
\text { especificaciones del } \\
\text { cliente/Total de obras } \\
\text { realizados }\end{array}$ & Mensual & $10 \%$ \\
\hline $\begin{array}{l}\text { Implementar } \\
\text { procesos de } \\
\text { innovación }\end{array}$ & $\begin{array}{l}\text { Realizar inspecciones } \\
\text { eficientes }\end{array}$ & $\begin{array}{l}\text { Desviación del } \\
\text { desperdicio } \\
\text { frente al } \\
\text { presupuesto }\end{array}$ & $\begin{array}{l}\text { (\% Desperdicio Real - } \\
\% \text { Desperdicio estándar) } \\
\text { 1\% Desperdicio estándar }\end{array}$ & Mensual & $2 \%$ \\
\hline $\begin{array}{l}\text { Aumentar la } \\
\text { productividad }\end{array}$ & $\begin{array}{l}\text { Realizar una ficha de } \\
\text { cumplimiento de obras } \\
\text { y su tiempo empleado }\end{array}$ & $\begin{array}{l}\text { Eficiencia del } \\
\text { manejo de } \\
\text { mano de obra }\end{array}$ & $\begin{array}{l}\text { (unidades producidas por } \\
\text { día hombre/unidades } \\
\text { programadas de } \\
\text { producción por día } \\
\text { hombre) } * 100\end{array}$ & Semanal & $91 \%$ \\
\hline
\end{tabular}

(a) 
ISSN: 2602-8085

\begin{tabular}{|c|c|c|c|c|c|}
\hline $\begin{array}{c}\text { Objetivo } \\
\text { Estratégico }\end{array}$ & $\begin{array}{c}\text { Forma de aplicación } \\
\text { del objetivo }\end{array}$ & Indicador & Método de Cálculo & Frecuencia & $\begin{array}{c}\text { Resultado } \\
\text { Esperado } \\
\%\end{array}$ \\
\hline $\begin{array}{l}\text { Aumentar la } \\
\text { productividad }\end{array}$ & $\begin{array}{l}\text { Realizar una ficha de } \\
\text { cumplimiento de } \\
\text { obras y su tiempo } \\
\text { empleado }\end{array}$ & $\begin{array}{l}\text { Eficiencia } \\
\text { del manejo } \\
\text { de mano de } \\
\text { obra }\end{array}$ & $\begin{array}{l}\text { (unidades producidas por } \\
\text { día hombre/unidades } \\
\text { programadas de } \\
\text { producción por día } \\
\text { hombre) } * 100\end{array}$ & Semanal & $91 \%$ \\
\hline $\begin{array}{l}\text { Mejorar los } \\
\text { procesos } \\
\text { continuamente }\end{array}$ & $\begin{array}{l}\text { Mejorar el proceso de } \\
\text { ensamblaje }\end{array}$ & $\begin{array}{l}\text { Costo por } \\
\text { resultado de } \\
\text { ensamblaje }\end{array}$ & $\begin{array}{l}\text { Costos totales/Producción } \\
\text { total }\end{array}$ & Mensual & $90 \%$ \\
\hline $\begin{array}{l}\text { Aumentar la } \\
\text { productividad }\end{array}$ & $\begin{array}{l}\text { Evitar presupuestos } \\
\text { rechazados }\end{array}$ & $\begin{array}{l}\text { Tasa de } \\
\text { construccio } \\
\text { nes }\end{array}$ & $\begin{array}{l}\text { Horas previstas para } \\
\text { inspecciones } \\
\text { planificadas/Total horas } \\
\text { disponibles }\end{array}$ & Mensual & $10 \%$ \\
\hline $\begin{array}{l}\text { Implementar el } \\
\text { área de gestión } \\
\text { estratégica }\end{array}$ & $\begin{array}{l}\text { Realizar compras de } \\
\text { materiales en base a } \\
\text { obras realizadas }\end{array}$ & $\begin{array}{l}\text { Compras de } \\
\text { materiales } \\
\text { efectuadas } \\
\text { dentro del } \\
\text { tiempo } \\
\text { programado }\end{array}$ & $\begin{array}{l}\text { Productos } \\
\text { entregados/Productos } \\
\text { programados x } 100\end{array}$ & Mensual & $20 \%$ \\
\hline $\begin{array}{l}\text { Mejorar el } \\
\text { mecanismo de } \\
\text { auditorías internas }\end{array}$ & $\begin{array}{l}\text { Realizar una } \\
\text { planificación de } \\
\text { auditorías internas }\end{array}$ & $\begin{array}{l}\text { Cumplimie } \\
\text { nto de } \\
\text { auditorías } \\
\text { internas }\end{array}$ & $\begin{array}{l}\text { Auditorías internas } \\
\text { realizadas/Auditorías } \\
\text { internas programadas }\end{array}$ & Semestral & $10 \%$ \\
\hline $\begin{array}{l}\text { Realizar el } \\
\text { manual de perfil } \\
\text { de competencias / } \\
\text { Elaborar un } \\
\text { sistema de } \\
\text { incentivos }\end{array}$ & $\begin{array}{l}\text { Realizar una ficha del } \\
\text { perfil de cada } \\
\text { trabajador y su } \\
\text { evolución con el } \\
\text { tiempo }\end{array}$ & $\begin{array}{l}\text { Nivel de } \\
\text { cumplimien } \\
\text { to }\end{array}$ & $\begin{array}{l}\text { (Puntos obtenidos por el } \\
\text { contenido/Puntos } \\
\text { requeridos por el perfil) } \\
* 100\end{array}$ & Anual & $10 \%$ \\
\hline $\begin{array}{l}\text { Implementar } \\
\text { cursos de } \\
\text { capacitación / } \\
\text { Desarrollar } \\
\text { conocimientos en } \\
\text { la gestión de } \\
\text { proyectos }\end{array}$ & $\begin{array}{l}\text { Realizar una } \\
\text { programación de } \\
\text { capacitación } \\
\text { identificando las } \\
\text { falencias existentes }\end{array}$ & $\begin{array}{l}\text { Capacitació } \\
\mathrm{n}\end{array}$ & $\begin{array}{l}\text { Número de empleados } \\
\text { capacitados/Total de } \\
\text { empleados inscritos*100 }\end{array}$ & Anual & $10 \%$ \\
\hline $\begin{array}{l}\text { Implementar el } \\
\text { Manual de } \\
\text { Seguridad } \\
\text { Industrial }\end{array}$ & $\begin{array}{l}\text { Socializar el manual } \\
\text { de seguridad } \\
\text { continuamente }\end{array}$ & $\begin{array}{l}\text { índice de } \\
\text { gravedad } \\
\text { SSO }\end{array}$ & $\begin{array}{l}\text { (días cargados + días } \\
\text { perdidos) x } 1000 \mathrm{k} / \text { horas } \\
\text { hombre trabajadas }\end{array}$ & Semestral & $10 \%$ \\
\hline
\end{tabular}

(b) Fuente: Implementación de objetivos estratégicos

\section{Resultados}

H0: El diseño de un Modelo de Gestión Estratégico no mejora la calidad y productividad de la empresa ORGATEC.

H1: El diseño de un Modelo de Gestión Estratégico mejora la calidad y productividad de la empresa ORGATEC. 
El nivel de significación será de 0,05 que es considerado para errores del tipo I, siendo la probabilidad de que se rechace la hipótesis nula.

De la tabla de distribución del Chi cuadrado con quince grados de libertad para 0.05 , el valor crítico es de 24,9958.

Tabla 8. Cuadro del cálculo del Chi cuadrado

\begin{tabular}{lrrr}
\hline \multicolumn{1}{c}{ INDICADOR } & REAL $(\mathbf{f 0})$ & META (fe) & (f0-fe) $^{\wedge} \mathbf{2} / \mathbf{f e}$ \\
\hline Eficiencia en ventas & 90,00 & 94,00 & 0,170 \\
Rotación del stock de ítems en inventario & 20,00 & 22,00 & 0,182 \\
Rechazo proveedores & 25,00 & 13,00 & 11,077 \\
Nivel de inventarios & 42,00 & 30,00 & 4,800 \\
Porcentaje de gastos financieros & 36,00 & 33,00 & 0,273 \\
Satisfacción del cliente & 4,00 & 5,00 & 0,200 \\
Producto conforme & 90,00 & 95,00 & 0,263 \\
Productos entregados de acuerdo con lo programado & 75,00 & 100,00 & 6,250 \\
Desviación de desperdicio frente a presupuesto & 2,00 & 1,50 & 0,167 \\
Eficiencia del manejo mano de obra & 89,00 & 91,00 & 0,044 \\
Costo por resultado de ensamblaje & 84,00 & 90,00 & 0,400 \\
Tasa de construcciones & 75,00 & 100,00 & 6,250 \\
Compras de materiales efectuadas dentro del tiempo & & & \\
programado & 20,00 & 25,00 & 1,000 \\
Cumplimiento de auditorías internas & 80,00 & 100,00 & 4,000 \\
Nivel de cumplimiento & 89,00 & 100,00 & 1,210 \\
Capacitación & 77,00 & 100,00 & 5,290 \\
& & & 41,575 \\
\hline
\end{tabular}

Fuente: Prueba estadística

El hecho de que acotemos la máxima probabilidad de error de tipo I (normalmente, por un valor próximo a cero, como 00,5 ) hace que difícilmente rechacemos $\mathrm{H} 0$ cuando no se debe; una consecuencia de esto es que las pruebas de hipótesis tienden a ser tremendamente conservadores con la hipótesis nula. (Horra, 2018). Para el cálculo de los grados de libertad se utilizará los valores obtenidos en el cuadro de mando integral, donde se tiene dos columnas una del valor real de los indicadores, otra los valores esperados y 16 filas. Por lo tanto, si se aplica el cálculo para determinar los grados de libertad se tiene que:

$$
\text { Grados de libertad }=(2-1)(16-1)=15
$$

El valor obtenido del Chi cuadrado es de 41,575 y comprado con el valor de la tabla que es de 26,296. Se observa que es mayor por lo tanto se rechaza la hipótesis nula y se acepta la 
hipótesis factible la cual establece que: El diseño de un Modelo de Gestión Estratégico mejora la calidad y productividad de la empresa ORGATEC.

\section{Conclusiones.}

Mediante la aplicación de encuestas a nivel estratégico, táctico, operativo y comercial, se determinó la situación actual del departamento de ingeniería, realizando un diagnóstico inicial de la empresa, el mismo que permitió establecer los procesos internos como son atención al cliente, ejecución de proyectos eléctricos, compras, ventas. Además de los procedimientos que conlleva a la realización de cada proceso, los mismos que deben ser mejorados debido a que provocan la insatisfacción en el cliente externo por la demora en los plazos de entrega.

Por medio de la elaboración de una matriz se obtuvo los indicadores claves de cada proceso, determinando los indicadores críticos a ser mejorados por medio del cuadro de mando integral que considera cuatro perspectivas, la financiera, cliente, procesos y de aprendizaje, planteando objetivos estratégicos para dar cumplimiento a la misión y visión establecidos por la administración de la empresa.

A través del enfoque basado en procesos, se clasifico a los procesos en tres tipos: procesos claves que son: ventas, compras, ensamblado, control de calidad; procesos estratégicos que son: atención al cliente, gestión financiera, gestión de recursos, gestión por procesos y los procesos de apoyo que son: gestión de calidad, capacitación, investigación y desarrollo.

Para el diseño del modelo de gestión se estableció estrategias las mismas que son: elaborar fichas de procesos para documentar la información obtenida, una matriz de resultados la misma que establece objetivos y procedimientos a seguir para conseguir el cumplimiento de las metas planteadas en cada indicador.

\section{Referencias bibliográficas.}

CT. Calidad. (2017). Norma de Control de Calidad y Norma de Revisión de Control de Calidad, 2a. ed. México: Instituto Mexicano de Contadores Públicos.

Borrego, A. (2014). La empresa española ante la crisis del modelo productivo: Productividad, competitividad e innovación. Bilbao: Fundación BBVA.

Salvio, F. (2015). Manual para iniciarse en la Calidad. México: Trillas.

Gutiérrez, E. (2014). Probabilidad y Estadística Aplicaciones a la Ingeniería y Ciencias, 1ra. ed. México: Grupo Editorial Patria.

Jiménez, R. (2015). Mantenimiento preventivo de sistemas de automatización industrial. Málaga: IC Editorial. 
Trashorras, J. (2016). Sistemas Eléctricos en Centrales. 1a. ed. España: Ediciones Paninfo SA. 2016.

Fonseca, R. (2015). Competitividad La clave del éxito empresarial. 1a.ed. Bogotá: Alfaomega Colombiana

Nardulli, G. (2015). Gestión organizacional. Buenos Aires: Editorial Maipue.

Toro, FJ. (2016). Costos ABC y presupuestos: herramientas para la productividad, 2a. ed. Bogotá: Ecoe Ediciones

Heizer, J. (2015). Dirección de la Producción y de Operaciones. 11a. ed. Madrid: Pearson Educación

Render, B. (2014). Principios de Administración de Operaciones. 9a.ed. México: Pearson Educación.

Horra, Jdl. (2018). Estadística aplicada, 3a. ed. Madrid: Ediciones Díaz de Santos, 2018

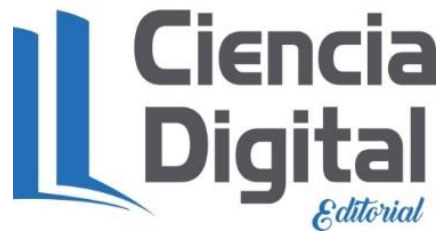




\section{PARA CITAR EL ARTÍCULO INDEXADO.}

Portalanza Molina, N., \& Valverde González, V. (2019). Diseño de un modelo de gestión estratégico para el mejoramiento de la productividad y calidad. Ciencia Digital, 3(3.2.1), 144-163. https://doi.org/10.33262/cienciadigital.v3i3.2.1.788

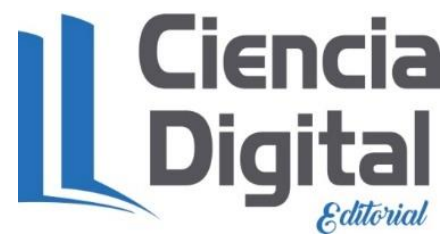

El artículo que se publica es de exclusiva responsabilidad de los autores y no necesariamente reflejan el pensamiento de la Revista Ciencia Digital.

El artículo queda en propiedad de la revista y, por tanto, su publicación parcial y/o total en otro medio tiene que ser autorizado por el director de la Revista Ciencia Digital.
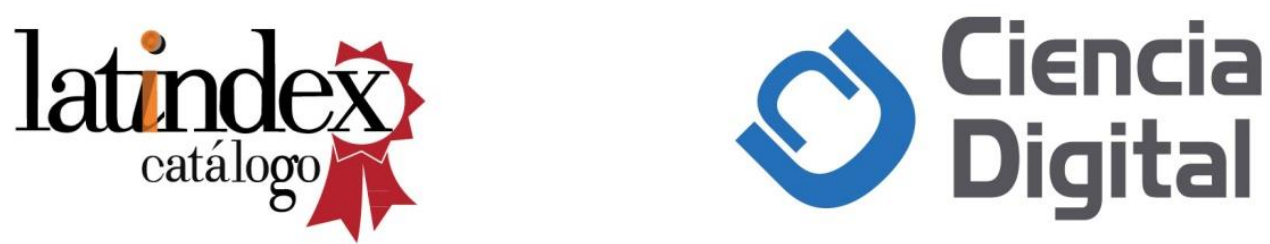